\title{
An Improved Method for Preparation of Uniform and Functional Mitochondria from Fresh Liver
}

\author{
Nagham Khouri Farah, Xiaocong Liu*, Catherine H. Wu and George Y. Wu \\ Department of Medicine, Division of Gastroenterology-Hepatology, University of Connecticut Health Center, Farmington, CT, USA
}

\begin{abstract}
Background and Aims: As the major energy source for mammalian cells, mitochondria have been the subject of numerous studies. However, the isolation and purification of healthy mitochondria, especially from fresh tissue, remains challenging. The most popular methods and kits involve various centrifugation steps which require substantial time and equipment but do not consistently provide pure preparations of functional mitochondria. The aim of this study was to determine whether methods could be devised to improve the purity and yield of functional mitochondria from fresh tissue. Methods: Fresh mouse liver was homogenized, and cells lysed. Particle size analysis, quantitation of mitochondrial DNA, mitochondrial oxygen consumption, and purity of mitochondria (by electron microscopy) were measured in samples after various purification steps and significant differences determined. Results: A two-step procedure consisting of centrifugation followed by filtration through $1.2 \mu$ and $0.8 \mu$ filters resulted in uniform mitochondrial preparations with diameters between 520-540 nm, and approximately 5 -times more pure samples. The mitochondria thus obtained had oxygen consumption and sensitivities to mitochondrial inhibitors that were indistinguishable from those purified by centrifugation alone. Electron microscopy confirmed the presence of more uniform and 4-5 times greater concentrations of mitochondria compared to centrifugation alone. Conclusions: A two-step procedure consisting of sequential centrifugation followed by filtration is a rapid method for the production of highly purified, uniform and functional mitochondria.
\end{abstract}

Citation of this article: Farah NK, Liu X, Wu CH, Wu GY. An improved method for preparation of uniform and functional mitochondria from fresh liver. J Clin Transl Hepatol 2019;7(1):46-50. doi: $10.14218 /$ JCTH.2018.00064.

\section{Introduction}

Mitochondria are the power-houses of almost all eukaryotic cells, generating about $90 \%$ of the chemical energy needed for cell viability. They also possess copies of mitochondrial specific DNA, which allow them to replicate as needed by the host cells. However, mitochondria are fragile organelles and

Keywords: Mitochondria; Isolation; Filtration; Purified; Uniform; Functional. Abbreviations: ADP, adenosine diphosphate; FCCP, carbonyl cyanide-4-(trifluoromethoxy)phenylhydrazone; IB, isolation buffer; qPCR, quantitative real time polymerase chain reaction.

Received: 14 December 2018; Accepted: 28 February 2019

* Correspondence to: Xiaocong Liu, Department of Medicine, Division of Gastroenterology-Hepatology, University of Connecticut Health Center, Farmington, CT 06030, USA. Tel: +1-860-679-2509, Fax: +860-679-6582, E-mail: xialiu@uchc.edu have specific intracellular requirements to function optimally. The host cell provides essential nutrients as well as proteins and transcripts encoded by the genomic DNA. Hence, the isolation and purification of healthy viable mitochondria have been challenging. The types of buffers used, and the time spent in the presence of those buffers have been shown to influence the quality of the mitochondrial preparations. For this reason, several kits have been produced containing proprietary buffers. In addition to the buffer systems, most methods to purify mitochondria rely on density gradient ultracentrifugation. However, this method requires specialized equipment, and involves long spin times, during which mitochondrial integrity can be compromised. Therefore, even with the use of commercial kits, the quality, viability and yield of mitochondria are variable. In the current study, we describe a rapid and efficient method to prepare mitochondria of a uniform size, with high levels of integrity and function.

\section{Methods}

\section{Mitochondrial isolation from mouse liver}

CD-1(ICR) mice of 21-30 days of age were anesthetized using ketamine/xylazine administered by intraperitoneal injection. The liver was removed and homogenized in the mitochondria isolation buffer (IB), as described by Frezza et al. ${ }^{1} \mathrm{~A} 100 \mathrm{~mL}$ solution of IB was prepared by adding $10 \mathrm{~mL}$ of $0.1 \mathrm{M}$ Tris-4-morpholinepropanesulfonic acid and $1 \mathrm{~mL}$ of $0.1 \mathrm{M}$ ethylene-bis(oxyethylenenitrilo)tetraacetic acid to 20 $\mathrm{mL}$ of $1 \mathrm{M}$ sucrose, adjusting the $\mathrm{pH}$ to 7.4 , and bringing the volume to $100 \mathrm{~mL}$ with distilled water. Protease inhibitor was added in a $1: 100$ (volume) ratio to IB.

The homogenate was transferred to $15 \mathrm{~mL}$ conical centrifuge tubes (USA Scientific, Orlando, FL, USA) and centrifuged at $1000 \times \mathrm{g}$ in an Allegra $\mathrm{X}-14 \mathrm{R}$ centrifuge (Beckman Coulter, Indianapolis, IN, USA) for $10 \mathrm{~m}$ at $4^{\circ} \mathrm{C}$, to pellet the nuclei, debris and large particles. The supernatant was subsequently transferred to $1.5 \mathrm{~mL}$ tubes (Eppendorf, San Diego, CA, USA) and centrifuged at $7,000 \times \mathrm{g}$ in the Eppendorf Centrifuge $5424 \mathrm{R}$ (Eppendorf) for $10 \mathrm{~m}$ at $4^{\circ} \mathrm{C}$. The supernatant was discarded; the pellet was resuspended in $100 \mu \mathrm{L}$ of ice-cold IB, transferred to a new $1.5 \mathrm{~mL}$ tube, and brought to $1 \mathrm{~mL}$ with IB. The suspension was centrifuged at $7,000 \times \mathrm{g}$ for $5 \mathrm{~m}$ at $4^{\circ} \mathrm{C}$ to eliminate small particles and debris. The supernatant was discarded, and pellets were combined and resuspended in $1 \mathrm{~mL}$ of IB. A final purification step consisted of sequential passage of the resuspended mitochondria through $1.2 \mu$ and $0.8 \mu$ pore filters using a $3-\mathrm{mL}$ syringe. The preparation was kept on ice and used within 1-3 $\mathrm{h}$ for measurement of mitochondrial DNA, size and charge, integrity and function. 


\section{Size analysis}

The size of isolated mitochondria was measured using a laser light scattering particle size analyzer (Model \#90 Plus; Brookhaven Instruments, Holtsville, NY, USA). Freshly prepared mitochondria were suspended in IB, and particle size measured.

\section{Quantitative real-time polymerase chain reaction ( $(P C R)$}

DNA was extracted from isolated mitochondria (DNeasy kit; Qiagen, Netherlands). Mitochondrial DNA (100 ng) was used as a template for qPCR with primers designed to amplify a specific region of mouse mitochondria at $116 \mathrm{bp}$ product length (primers: 5'-TCGCCTACTCCTCAGTTAGCCACA-3', 5'-TGATGATGTGAGGCCATGTGCGA-3'; Integrated DNA Technology, Skokie, IL, USA). $\Delta \Delta$ Ct values were calculated to compare the mitochondrial DNA to lactate dehydrogenase $A$ signals as an indication of nuclear DNA contamination (primers: $5^{\prime}$-TAATGAAGGACTTGGCAGATGAACT-3', 5'-ACGGCTTTCTCCCTCTTGCT-3'; Integrated DNA Technology). Copy number was determined by preparation of a specific sequence of mouse mitochondrial DNA (5'-TCGCCTACTCCTCAGTTAGCCACATAGCACTTGTTATTGCATCAATCATAATCCAAACTCCATGAAGCTTCATAGGAGCAACAATACTAATAATCGCACATGGCCTCACATCATCA-3') by amplification, which was then used to generate a standard curve from which quantities of experimental samples could be determined.

\section{Cytochrome C assay}

Integrity of the mitochondrial outer membrane was determined by cytochrome c oxidase assay kit (Sigma-Aldrich, St. Louis, MO, USA), as instructed by the manufacturer. In brief, samples of freshly prepared mitochondria were suspended in $0.2 \mathrm{mg} / \mathrm{mL}$ in enzyme dilution buffer containing $1 \mathrm{mM}$ $\mathrm{n}$-dodecyl $\beta$-D-maltoside (Sigma-Aldrich), as a detergent. A control sample was suspended in $0.2 \mathrm{mg} / \mathrm{mL}$ in enzyme dilution buffer alone. Samples were incubated at $4 \mathrm{C}$ for $10 \mathrm{~m}$, and then $20 \mu \mathrm{L}$ of each sample was added to cuvettes containing $950 \mu \mathrm{L}$ of assay buffer $(10 \mathrm{mM}$ Tris- $\mathrm{HCl}, 120 \mathrm{mM}$ $\mathrm{KCl}, \mathrm{pH} \mathrm{7.0)}$ and $80 \mu \mathrm{L}$ of enzyme dilution buffer. The cuvettes were placed into a spectrophotometer. The assay was started by adding $50 \mu \mathrm{L}$ of ferrocytochrome c substrate (SigmaAldrich) (0.22 mM, reduced by dithiothreitol at a final concentration of $0.5 \mathrm{mM}$ ) to cuvettes and mixing by pipetting. Absorbance $\left(A^{550}\right)$ was read at $5,15,25,35$ and 45 s after the addition of ferrocytochrome $c$ substrate. A graph of $A^{550}$ as a function of time was plotted to calculate the maximum linear rate $(\mathrm{A} / \mathrm{s})$ for both sample and control. The degree of mitochondrial integrity was calculated by the equation below:

\section{\%lactate dehydrogenase $\mathbf{A}=$ $[\Delta \mathbf{A} / \mathbf{s}$ (with detergent $)-\Delta \mathbf{A} / \mathbf{s}($ without detergent $)] /$ $[\Delta \mathbf{A} / \mathbf{s}($ with detergent $)] \times \mathbf{1 0 0} \%$}

$$
\Delta \mathbf{A} / \mathbf{s}=\mathbf{A} / \mathbf{s}(\text { sample })-\mathbf{A} / \mathbf{s}(\text { blank })
$$

\section{Transmission electron microscopy}

Samples of mitochondria from before and after filtration were fixed with glutaraldehyde $2.5 \%$, stained with uranyl acetate
(Sigma-Aldrich), embedded in plastic, sectioned (Ultramicrotome Leica Electron Microscope UC7; Leica Biosystems, Buffalo Grove, IL, USA) and examined by transmission electron microscopy on a Hitachi H-7650 transmission electron microscope (Tokyo, Japan). The numbers of intact mitochondria were quantitated per field using Imagej.exe and expressed as means \pm standard deviations per field at identical magnifications.

\section{Respiration assay}

The respiration mitochondria as determined by oxygen consumption were assayed using the Seahorse Analyzer XF24 (Seahorse Bioscience, North Billerica, MA, USA) as described in the Seahorse isolated mitochondria respiration assay protocol. Mitochondria, $8 \mu \mathrm{g}$ measured by BCA protein assay (Pierce, Rockford, IL, USA) were placed in each well, and exposed sequentially to adenosine diphosphate (ADP), oligomycin, carbonyl cyanide-4-(trifluoromethoxy)phenylhydrazone (FCCP), and antimycin. Oxygen consumption was expressed in units of pmole/m normalized to the amount of mitochondrial DNA present as determined by GPCR and expressed as means \pm standard deviations.

\section{Results}

Table 1 shows laser light scattering data on particle size and charge at various stages of mitochondrial preparation. The mean particle diameter from after the low speed was $295 \mathrm{~nm}$, reflecting elimination of large impurities. After the first and second high-speed spins, the mean particle diameters increased to $409 \mathrm{~nm}$ and $566 \mathrm{~nm}$, respectively, reflecting elimination of small debris. Electron microscopy images of mitochondria preparations after high-speed spins still showed considerable evidence of membrane fragments. For this reason, the effects of filtration were studied. Table 1 shows that filtration through $1.2 \mu$ and $0.8 \mu$ membranes progressively decreased mean particle diameter to $536 \mathrm{~nm}$ and $520 \mathrm{~nm}$, respectively.

\section{Mitochondrial DNA}

To quantitate the effects of the purification steps, mitochondrial DNA was measured by quantitative PCR analysis by amplifying a specific region of 116 bp relative to lactate dehydrogenase $A$, which is a marker of genomic DNA contamination, and those sequences are absent in mitochondrial DNA. As shown in Table 1, mitochondrial DNA was significantly purer after each step of filtration. The highand low-speed spins resulted in a 1.7-fold increase in mitochondrial DNA over genomic contamination. The additional filtration steps increased the ratio 6.7 -fold. To provide a better comparison in terms of amount of mitochondrial DNA at each step, the mitochondrial to LDH copy number ratio increased from 1200 to 8000 after that last filtration step. The data indicate that the sequential filtration steps significantly improve the mitochondrial purity compared to the standard method of varying speed spins alone.

\section{Integrity of the mitochondria outer membrane}

In order to assess if the mitochondria were intact after passing the filters, cytochrome $\mathrm{C}$ activity assay was used to test for the integrity of the mitochondria outer membrane. The values obtained showed that $95 \pm 3.1 \%$ of the isolated mitochondria remained intact after the final filtration step. 
Farah N.K. et al: Mitochondria isolation and purification

Table 1. Particle size and mitochondrial DNA data at various steps in mitochondrial purification

\begin{tabular}{|c|c|c|c|}
\hline Purification Step & Particle Size in nm & $\begin{array}{l}\text { Mitochondrial DNA as } \\
\text { fold-change/LDHA }\end{array}$ & $\begin{array}{l}\text { Mitochondrial DNA as mitochondrial } \\
\text { copy number/LDHA }\end{array}$ \\
\hline $1000 \times g$ spin: Supernatant & $300 \pm 10$ & 1.0 & $1200 \pm 270$ \\
\hline First $7000 \times g$ spin: Pellet & $410 \pm 10$ & 1.3 & $1590 \pm 390$ \\
\hline Second $7000 \times$ g spin: Pellet & $570 \pm 20$ & 1.7 & $1990 \pm 420$ \\
\hline Filtrate: $1.2 \mu$ & $540 \pm 20$ & 4.2 & $5050 \pm 1400$ \\
\hline Filtrate: $0.8 \mu$ & $520 \pm 10$ & 6.7 & $8010 \pm 1590$ \\
\hline
\end{tabular}

Abbreviation: LDHA, lactate dehydrogenase A.

\section{Mitochondrial function}

The cytochrome assay showed that the isolated mitochondria remained intact after filtration. However, other damage could have occurred during the purification steps, especially after the pressure applied during the sequential filtration steps. To determine whether or not the filtration resulted in signification changes in mitochondrial function, measurement of mitochondrial respiration function was undertaken. Fig. 1 shows that respiration mitochondria after the standard high-speed spin prep was indistinguishable from that of mitochondria purified by sequential filtration. In particular, in mitochondria before filtration, activation by ADP increased the oxygen consumption rate from 170 to $530 \mathrm{pmol} / \mathrm{m}$. Similarly, for mitochondria after spins and dual sequential filtration, activation by ADP increased oxygen consumption rate from 180 to $540 \mathrm{pmol} / \mathrm{m}$. Oligomycin decreased the oxygen consumption rate for mitochondria from high-speed spins alone and spins plus filtration by about $30 \%$. Release by FCCP and final inactivation by antimycin, all characteristic of mitochondrial oxygen consumption, were not significantly different. The data suggest that the mitochondrial samples retained their energy generating function, and the filtrations did not significantly alter mitochondrial respiratory function.

\section{Electron microscopy}

Electron micrograph analysis was performed to determine whether there were visible differences in the mitochondrial preparations purified by standard high-speed spins alone (before filtration), and those after spins and sequential centrifugation. Fig. 2, left panel shows that at $5000 \times$ there was a considerable amount of large and small membranous debris in the filtered mitochondria compared to the mitochondria before filtration. In contrast, there was much less membranous debris after filtrations (Fig. 2, middle panel). Furthermore, as seen in Fig. 2, right panel, the mean number of mitochondria identified per field after spins alone was 17 , while the mean number of mitochondria per field was measured as 50. This difference between preparations only centrifuged compared to sequential centrifugation and filtration was significantly different.

\section{Discussion}

There have been many different methods published on the preparation and isolation of mitochondria. ${ }^{1-4}$ There are also several commercial kits available for this purpose (e.g., mitochondria isolation kit by Thermo Scientific, MITOISO2 by Sigma, and Qproteome by Qiagen). ${ }^{5-8}$ The reason for the diversity of

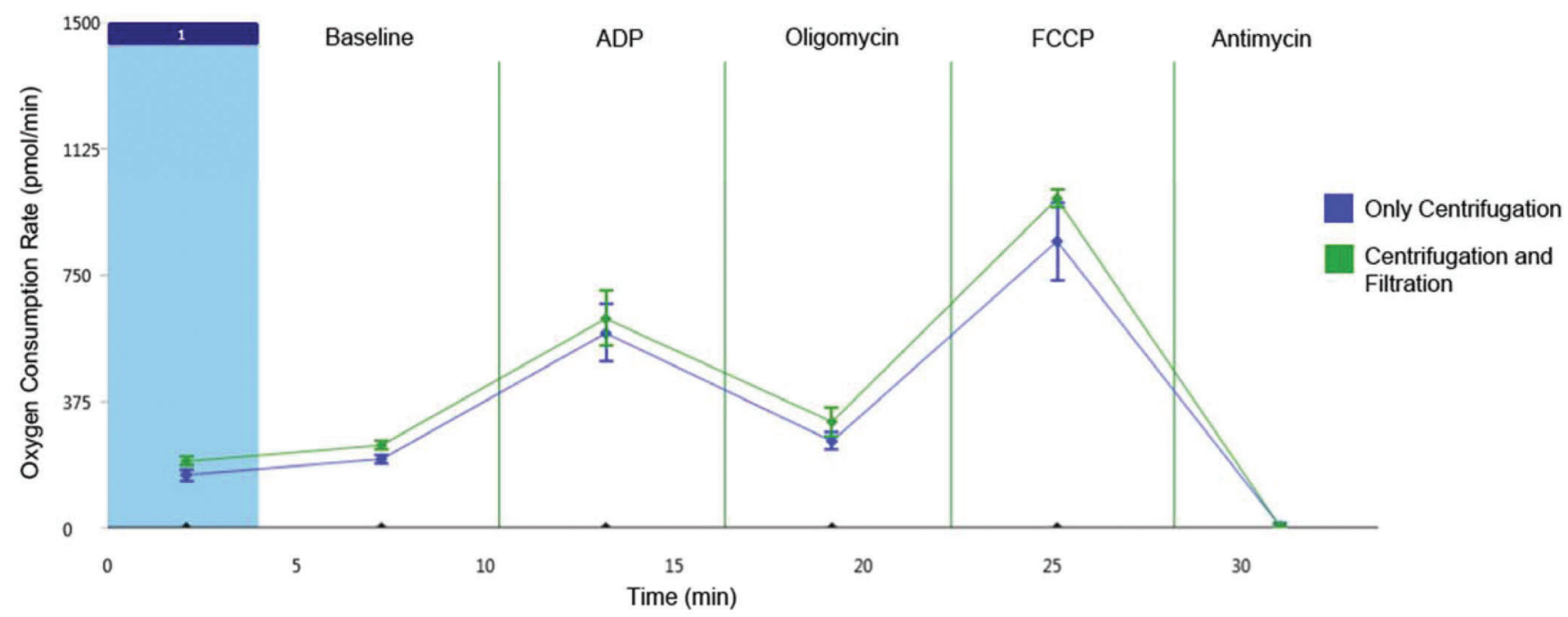

Fig. 1. Oxygen consumption. The data are expressed in units of pmol/m normalized to the amount of mitochondrial DNA present as determined by $q P C R$ and expressed as means \pm standard deviation.

Abbreviation: qPCR, quantitative real time polymerase chain reaction. 


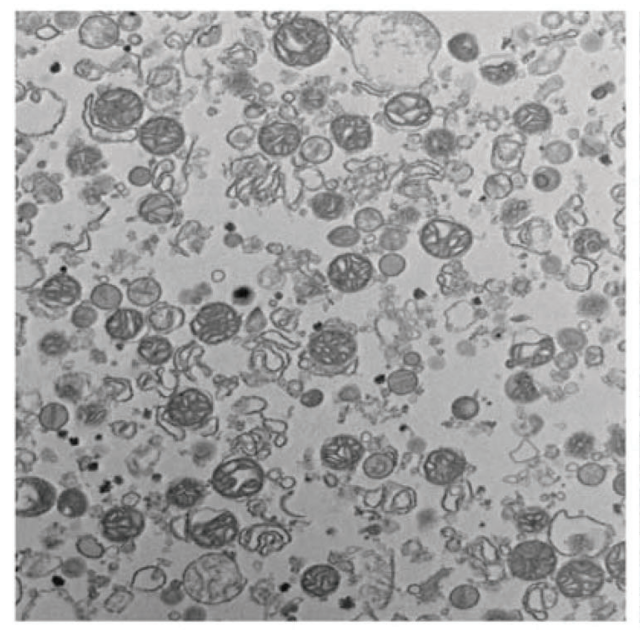

Only Centrifugation

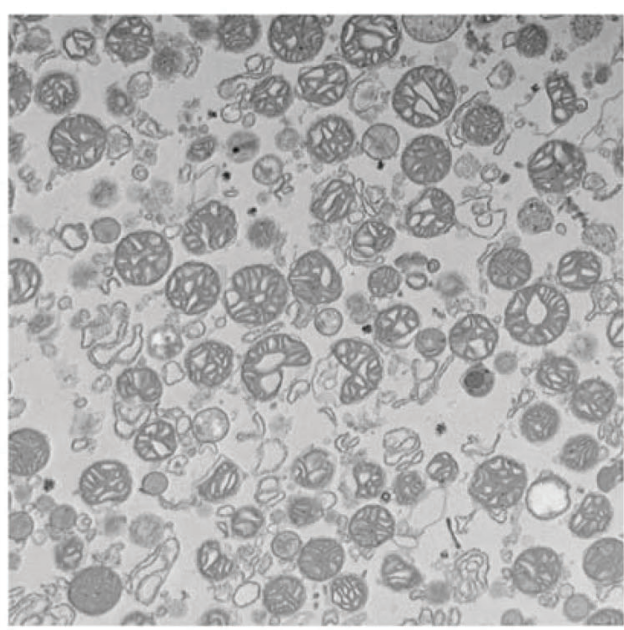

Centrifugation and Filtration

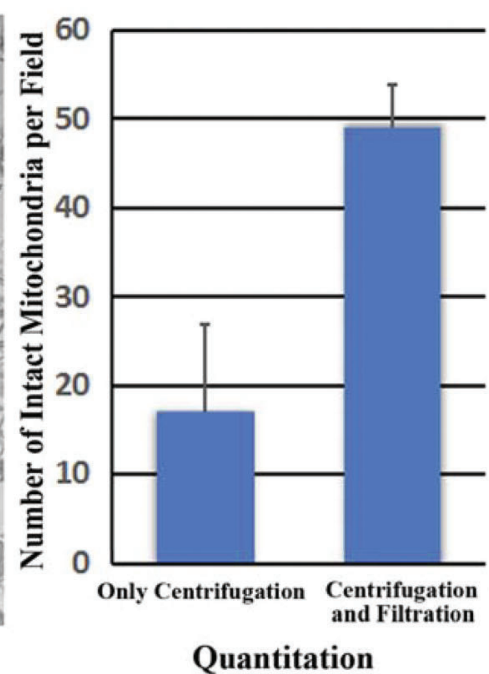

Quantitation

Fig. 2. Quantitative effects of filtration on purity of mitochondrial preparation purity. Determined by transmission electron microscopy (per microscopic field at $5000 \times)$.

methods lies in the fragility of the membranous structures, and sensitivity to changes in $\mathrm{pH}$, osmolality, temperature, and ionic concentrations. This is especially true for isolation of mitochondria from tissues, where homogenization is required before the isolation process. ${ }^{9}$ Indeed, there are separate and general simpler protocols for isolation of mitochondria from cultured cells. ${ }^{10}$ For final purification, equilibrium density gradient ultracentrifugation is probably the most popular technique. However, this method requires special equipment and considerable time for the mitochondria to reach their density. This is usually not a problem for structural studies but can be a problem for functional studies. Variability in the quality, viability, and yield of mitochondria have been reported. ${ }^{11}$

Besides equilibrium density gradient ultracentrifugation, other physical methods have been studied to improve the purity of mitochondrial preparations. For example, Banik and Dhar $^{12}$ isolated functional mitochondria using paramagnetic iron oxide nanoparticles. Although purification was achieved, the nanoparticles could not be separated from the mitochondria after isolation, which might limit the value of this method. Free-flow zonal electrophoresis was also used to purify mitochondria, ${ }^{13}$ which requires a special free-flow apparatus and considerable time for preparation.

We found that low- and high-speed spin steps based on the method previously described by Frezza et al. ${ }^{1}$ provided good initial removal of cellular debris without significantly altering mitochondrial function (data not shown). Filtration has been described previously to eliminate relatively large particulate matter from mitochondrial preparations. For example, Preble et al. ${ }^{14}$ used filtration through sequential $40 \mu$ and $10 \mu$ nylon filters, followed by a high-speed spin for final purification of mitochondria from fresh muscle tissue. ${ }^{14}$ Because the mean diameter of mitochondria has been reported to be between 500 and $1000 \mathrm{~nm}$, these membrane filters allow mitochondria to pass though while removing large debris such as incompletely lysed cells and large organelle aggregates.

Our selection of the much more restrictive $1.2 \mu$ and $0.8 \mu$ filters was designed to increase the purity of preparations by maximizing the elimination of debris larger than mitochondria. Indeed, some large mitochondria were doubtless also lost during our preparation. However, because we were interested in obtaining not only the purest but the smallest and most uniform populations of mitochondria, these filters served our purposes best. If uniformly small mitochondria are not needed, a single filtration through $1.2 \mu$ filter would improve the yield. Nevertheless, this is the first description of a mitochondrial isolation method using simple rapid spin steps and a sequential sub-1.2 $\mu$ filtration to provide isolated uniform highly purified and functional mitochondria from liver tissue.

We conclude that a two-step procedure consisting of sequential centrifugation followed by membrane filtration results in preparations of highly purified, uniform, and functional mitochondria, and represents a significant improvement in the preparation of mitochondria from fresh liver tissue.

\section{Acknowledgments}

The support of the Herman Lopata Chair in Hepatitis Research is gratefully acknowledged. We thank Dr. Vjera Nincevic for her technical assistance.

\section{Conflict of interest}

The authors have no conflict of interests related to this publication.

\section{Author contributions}

Carried out most of the mitochondrial isolation and purification experiments (NKF), did the qPCR and oxygen consumption measurements, and wrote the results section $(X L)$, advised on mitochondrial isolation ( $\mathrm{CHW}$ ), and designed the experiments and edited the manuscript (GYW).

\section{References}

[1] Frezza C, Cipolat S, Scorrano L. Organelle isolation: functional mitochondria from mouse liver, muscle and cultured fibroblasts. Nat Protoc 2007;2:287295. doi: $10.1038 /$ nprot.2006.478. 
[2] Hogeboom GH, Schneider WC, Pallade GE. Cytochemical studies of mammalian tissues; isolation of intact mitochondria from rat liver; some biochemical properties of mitochondria and submicroscopic particulate material. J Biol Chem 1948;172:619-635.

[3] Djafarzadeh S, Jakob SM. Isolation of intact mitochondria from skeletal muscle by differential centrifugation for high-resolution respirometry measurements. J Vis Exp 2017. doi: 10.3791/55251.

[4] Renault TT, Luna-Vargas MP, Chipuk JE. Mouse Liver Mitochondria Isolation, Size Fractionation, and Real-time MOMP Measurement. Bio Protoc 2016;6: e1892. doi: 10.21769/BioProtoc.1892.

[5] Witter RF, Watson ML, Cottone MA. Morphology and ATP-ase of isolated mitochondria. J Biophys Biochem Cytol 1955:1:127-138, doi: 10.1083/jcb.1.2.127.

[6] Jackson $\mathrm{KL}$, Pace $\mathrm{N}$. Some permeability properties of isolated rat liver cell mitochondria. J Gen Physiol 1956;40:47-71. doi: 10.1085/jgp.40.1.47.

[7] Tedeschi H. Some observations on the permeability of mitochondria to sucrose. J Cell Biol 1965;25:229-242. doi: 10.1083/jcb.25.2.229.

[8] Stoner CD, Sirak HD. Osmotically-induced alterations in volume and ultrastructure of mitochondria isolated from rat liver and bovine heart. J Cell Biol 1969;43:521-538. doi: 10.1083/jcb.43.3.521.

[9] Schmitt $S$, Eberhagen $C$, Weber $S$, Aichler $M$, Zischka $H$. Isolation of mitochondria from cultured cells and liver tissue biopsies for molecular and biochemical analyses. Methods Mol Biol 2015;1295:87-97. doi: 10 . 1007/978-1-4939-2550-6_8.

[10] Wettmarshausen J, Perocchi F. Isolation of functional mitochondria from cultured cells and mouse tissues. Methods Mol Biol 2017;1567:15-32. doi: 10 1007/978-1-4939-6824-4_2.

[11] Afanasyeva MA, Ustiugova AS, Golyshev SA, Kopylov AT, Bogolyubova AV Demin $\mathrm{DE}$, et al. Isolation of large amounts of highly pure mitochondria for "omics" studies. Biochemistry (Mosc) 2018;83:76-85. doi: 10 1134/S0006297918010108.

[12] Banik B, Dhar S. Centrifugation-free magnetic isolation of functional mitochondria using paramagnetic iron oxide nanoparticles. Curr Protoc Cell Biol 2017;76:25.4.1-25.4.20. doi: 10.1002/cpcb.26.

[13] Zischka $H$, Lichtmannegger J, Jägemann $N$, Jennen $L$, Hamöller $D$, Huber $E_{\text {, }}$ et al. Isolation of highly pure rat liver mitochondria with the aid of zoneelectrophoresis in a free flow device (ZE-FFE). Methods Mol Biol 2008;424 333-348. doi: 10.1007/978-1-60327-064-9 26.

[14] Preble JM, Pacak CA, Kondo H, MacKay AA, Cowan DB, McCully JD. Rapid isolation and purification of mitochondria for transplantation by tissue dissociation and differential filtration. J Vis Exp 2014:e51682. doi: 10 $3791 / 51682$. 